\title{
Editorial
}

\section{Pacing for vasovagal syncope}

In most cases vasovagal syncope is an infrequent event that only merits reassurance. However, in a few patients the symptoms can be debilitating, resulting in actual physical injury or considerable infringement of daily living and work. In these cases, a more interventional approach may be appropriate.

\section{Evidence for the efficacy of pacing}

The vasovagal response is a notable but transient dysfunction of the autonomic nervous system. The most readily quantitative responses are vasodepression (arterial blood pressure fall) and cardioinhibition (heart rate fall). Cardiac pacing offers the possibility specifically to target the latter. Evidence to support the use of pacing in this setting has recently gained additional weight with the publication of the vasovagal pacemaker study. ${ }^{1}$ Patients with recurrent episodes of vasovagal syncope (more than six each year) were randomised to receive or not receive a permanent dual chamber pacemaker. This important study indicated a clear benefit for pacing in those with vasovagal syncope selected on the basis of positive tilt test with bradycardia. In those allocated to receive a pacemaker there was a notable extension in the time to recurrence of syncope with a relative risk reduction of $85.4 \%$. This treatment effect remained despite adjusting for the observed baseline differences between the paced and non-paced groups (relative risk reduction of $90.8 \%$ ).

Even before the vasovagal pacemaker study ${ }^{1}$ cardiac pacing has been an accepted treatment for selected patients with vasovagal syncope by both the British Pacing Electrophysiology Group ${ }^{2}$ and American Heart Association/ American College of Cardiology ${ }^{3}$ guidelines; these recommendations were based on the results of several nonrandomised observational studies. Despite some exceptions, ${ }^{4}{ }^{5}$ these studies generally indicated a beneficial role for pacing. ${ }^{6-8}$ The inconsistency of these studies may be in the interpretation of the results rather than the results themselves. Opponents point to the inability of pacing consistently to prevent syncope; proponents point to the prolongation of the time to syncope even if syncope is not aborted. Consequently, studies such as that of Sra and colleagues $^{5}$ can be interpreted as negative because presyncope and syncope are not invariably aborted, or more positively because even their simple pacing algorithm resulted in many patients (18 of 22) who initially had syncope had only presyncope on repeat testing.

\section{Mode of pacemaker intervention}

Concomitant with the accumulation of data supporting the use of pacing in vasovagal syncope, there has been a better understanding of appropriate pacemaker settings. Initial studies showed that ventricular demand pacing (VVI) usually fails to ameliorate symptoms even if a bradycardiac response prevails. ${ }^{9}$ The absence of atrioventricular synchrony appears to aggravate the peripheral vasodilatation, perhaps by retrograde activation of atria and release of natriuretic peptides, which generally accompanies this condition. The problem of early detection of incipient vasovagal syncope remains. Unlike other conditions requiring pacing, the fall in heart rate is not abrupt. More often the decline is insidious. DDD pacing with rate drop response appears to confer an advantage over the more conventional DDI pacing with rate hysteresis. ${ }^{10}$ As opposed to rate hysteresis, which triggers pacing when a particular heart rate is reached, rate drop response takes account of the rate of fall and has a requirement for confirmatory beats. Hence, the incorporation of this more sophisticated type of algorithm offers an additional option in the appropriate detection of the heart rate changes, facilitating more appropriate and earlier triggering of pacing. Further refinement in the ability to detect incipient vasovagal syncope may arise from the recognition of the accompanying respiration changes. ${ }^{11}$

\section{Determining who benefits from pacing}

The crucial issue is to identify people who could benefit from pacing. The most frequently used investigation is the tilt test. The vasovagal pacemaker study ${ }^{1}$ criteria were the occurrence of at least syncopal episodes and a positive tilt test with a relative bradycardia (that is, trough heart rate less than 60 beats/min or less than $70-80$ beats/min if isoprenaline provocation was used).

A systematic classification based on haemodynamic collapse pattern for the purposes of delineating patients who might be candidates for drug or pacemaker trials for vasovagal syncope treatment has been proposed by the vasovagal syncope international study (VASIS) group. ${ }^{12}$ There are three major VASIS categories: predominant cardioinhibition, predominant vasodepression, and mixed cardioinhibition and vasodepression. In addition there are three exceptions: chronotropic incompetence, excessive heart rate rise, and carotid sinus syndrome. Those with predominant cardioinhibition might be suitable for pacing. Within this group there is further division into more or less severe forms. The expectation is that pacing will be of greatest benefit to those with more severe cardioinhibition. However, there is a potential concern with pacing this group as it appears to be more prevalent in the younger population ${ }^{13}$; (mean age of patients enrolled in the vasovagal pacemaker study was $43^{1}$ ). Pacing young people has a considerable long term burden, not least of which is the need for periodic system replacement. Given that vasovagal syncope is not in itself a life threatening condition there is a natural reluctance to commit younger patients to pacing.

The other group that might benefit from pacing are those with chronotropic incompetence (VASIS exception 1). ${ }^{14}$ They tend to be a much older population and without tilt testing may remain unidentified. They may be physically unable to undergo exercise testing, or be unsuitable for pharmacological testing owing to comorbidity such as coronary artery disease.

Compensating for the decline in heart rate only deals with one aspect of the underlying pathophysiological changes. Pacing should not be seen in isolation or as an alternative to pharmacological intervention. There may be a role for combined treatment-for example, with drugs such as midodrine ${ }^{15}$ to modulate vascular tone or fludrocortisone to minimise intravascular volume depletion and pacing to modify the heart rate response. With greater understanding of the underlying pathophysiology the appropriate treatment may be tailored to the individual patient. 
There are limitations to the benefits of pacing in vasovagal syncope. Clearly it does not abolish syncope in all patients. However, the consistent finding is the potential for delaying loss of consciousness. The additional time gained may be sufficient for the patient to take evasive action, preventing syncope and injury to themselves or others. In a small number of selected patients with severe vasovagal symptoms with a notable bradycardiac response on tilt testing, dual chamber pacing with an appropriate rate drop algorithm may be a reasonable therapeutic option.

Department of Cardiology, Imperial College School of Medicine,

A S KURBAAN St Mary's Hospital, Praed Street, London W2 1NY, UK

R SUTTON

Department of Cardiology, Imperial College School of Medicine,

Royal Brompton Hospital, Sydney Street, London SW3 6NP, UK

1 Connolly SJ, Sheldon R, Roberts RS, et al, on behalf of the Vasovagal Pacemaker Study Investigators. The North American vasovagal pacemaker study (VPS). A randomised trial of permanent cardiac pacing for the prevention of vasovagal syncope. $\mathcal{F}$ Am Coll Cardiol 1999;33:16-20.

2 Clarke M, Sutton R, Ward D, et al. British Pacing and Electrophysiology Group Working Party. Recommendations for pacemaker prescription for symptomatic bradycardia. Br Heart f 1991;66:185-91.

3 Gregartos G, Cheitlin MD, Conill A, et al. ACC/AHA guidelines for implantation of cardiac pacemakers and antiarrhythmia devices: executive summary. A report of the American College of Cardiology/American Heart Association Task Force on Practice Guidelines (Committee on Pacemaker Implantation). Circulation 1998;97:1325-35.
4 El-Bedawi KM, Wahba MMAE, Hainsworth R. Cardiac pacing does not improve orthostatic tolerance in patients with vasovagal syncope. Clin Auton Res 1995;4:233-7.

5 Sra JS, Jazayeri MR, Avitall B, et al. Comparison of cardiac pacing with drug therapy in the treatment of neurocardiogenic (vasovagal) syncope with bradycardia or asystole. N Engl F Med 1993;328:1085-90.

6 Fitzpatrick A, Theodorakis G, Ahmed R, et al. Dual chamber pacing aborts vasovagal syncope induced by head-up 60 degree tilt. PACE 1991;14:1319.

7 Samoil D, Grubb BP, Brewster P, et al. Comparison of single- and dual chamber pacing techniques in prevention of upright tilt-induced vasovagal syncope. Eur 7 Cardiac Pacing Electrophysiol 1993;3:36-41.

8 Petersen MEV, Chamberlain-Webber R, Fitzpatrick AP, et al. Permanent pacing for cardio-inhibitory malignant vasovagal syndrome. Br Heart $\mathcal{F}$ 1994;71:274-81.

9 Fitzpatrick AP, Travill CM, Vardas PE, et al. Recurrent symptoms after ventricular pacing in unexplained syncope. PACE 1990;13:619-24.

10 Ammirati F, Colivicchi F, Toscano S, et al. DDD pacing with rate drop function response versus DDI with rate hysteresis for cardioinhibitory vasovagal syncope. PACE 1998;21):2178-81.

11 Lipsitz LA, Hayano J, Sakato S, et al. Complex demodulation of cardiorespiratory dynamics preceding vasovagal syncope. Circulation 1998;98:97783.

12 Sutton R, Petersen M, Brignole M, et al. Proposed classification for vasovagal syncope. Eur f Cardiac Pacing Electrophysiol 1992;3:180-3.

13 Kurbaan AS, Franzén A-C, Bowker TJ, et al. Usefulness of tilt induced patterns of heart rate and blood pressure using a two stage protocol with glyceryl trinitrate provocation in patients with syncope of unknown origin. $\mathrm{Am}$ 7 Cardiol 1999;84:665-70.

14 Kurbaan AS, Franzén A-C, Williams TR, et al. Chronotropic incompetence in the older population with syncope can be identified using tilt testing with nitrate provocation [abstract]. PACE 1999;22 (4 part II):459.

15 Ward CR, Gray JC, Gilroy JJ, et al. Midodrine: a role in the management of neurocardiogenic syncope. Heart 1998;79:45-9.

\section{STAMPS IN CARDIOLOGY}

\section{Conferences}

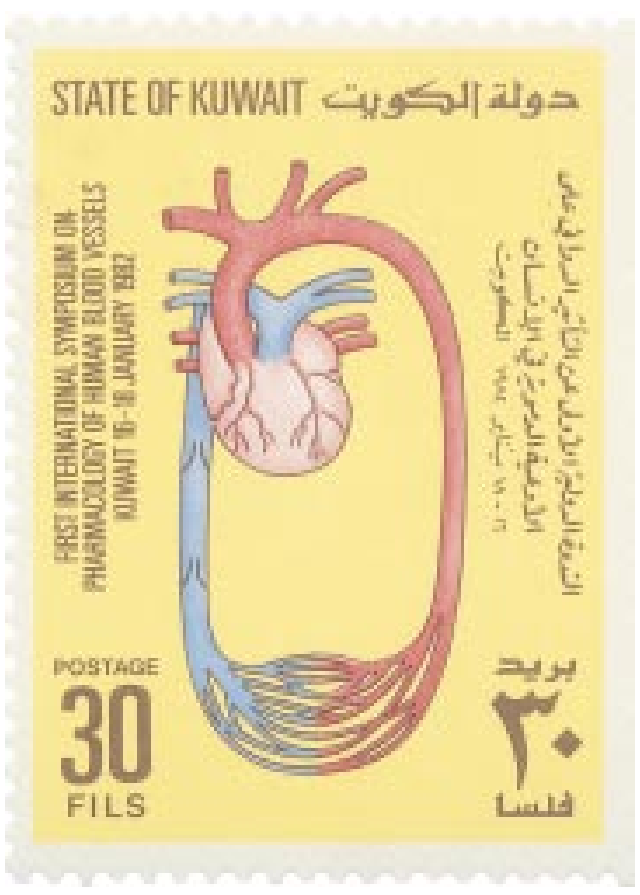

This very well designed stamp from Kuwait, depicting the anatomical heart and the large and small vasculature of the venous and arterial circulation, was issued in 1982 in recognition of the First International Symposium on Pharmacology of Human Blood Vessels held in Kuwait on 16-18 January that year.

$M$ K DAVIES A HOLLMAN 